\section{Modellvorhaben zur Qualitätssicherung in der Phytotherapie - Interview}

Die BARMER Ersatzkasse hat in Zusammenarbeit mit dem Bundesverband der pharmazeutischen Industrie (BPI) im Sommer 1998 die Planung für ein Modellvorhaben «zur Förderung der Verordnung von pflanzlichen Arzneimitteln, Homöopathika und anthroposophischen Arzneimitteln gemäß SGB V» gestartet. Als erste Stufe dieses Vorhabens wurden mit Hilfe einer neunköpfigen Expertenkommission unter Vorsitz von Prof. Dr. med. Volker Fintelmann, Hamburg, für einzelne Heilpflanzen Kriterien erarbeitet, die Präparate aus diesen Drogen jeweils erfüllen müssen, wenn sie im Rahmen dieses Modellvorhabens verordnungsfähig sein sollen. «Es war nicht erklärtes Ziel, sämtliche Indikationen und die dazugehörigen Drogen zu bewerten, sondern einen beispielhaften Ausschnitt einer rationalen Phytotherapie aufzuzeigen», vermerkt die Kommission dazu. Für die wichtigsten Drogenextrakte entstand so ein konkreter Katalog von Qualitätsanforderungen, die den aktuellen Stand des Wissens widerspiegeln und einen beispielhaften Ausschnitt für eine rationale Phytotherapie darstellen.

Wie intensiv zur Zeit die Bemühungen der Forschung sind, im Bereich der Phytotherapie einheitliche Qualitätsstandards zu etablieren, zeigt auch die in diesem Jahr erfolgte Gründung des «Komitee Forschung Naturmedizin (KFN)». Im Rahmen dieser Arbeitsgemeinschaft werden unter anderem für die wichtigsten Drogen bzw. Indikationen jeweils KFN-Dokumentationen erstellt, die im Sinne einer EBM die Qualität und die Wirksamkeit der Phytopharmaka durch wissenschaftliche Veröffentlichungen belegen.

FORSCHENDE KOMPLEMENTÄRMEDIZIN sprach mit dem Initiator des Modellvorhabens, Dr. Gerd Glaeske, zum Zeitpunkt des Interviews Leiter der Abteilung medizinisch wissenschaftliche Grundsatzfragen der BARMER Ersatzkasse, Wuppertal, der im wissenschaftlichen Beirat des KFN mitarbeitet.

Was war die Motivation für dieses Vorhaben?

Glaeske: Unsere Motivation geht auf zwei Gründe zurück: Der eine ist, dass der Markt der pflanzlichen Präparate in Deutschland immer noch sehr unübersichtlich ist. Dadurch dass parallel zugelassene und bislang nicht zugelassene Arzneimittel nebeneinander existieren, nur unterschieden durch den Packungsaufdruck «Reg. Nr. ...» bzw. «Zul. Nr. ...», ist für Ärzte keine ausreichende Transparenz gegeben. Auch sie können qualitativ gute Präparate von Mitteln mit einer zweifelhaften Qualität nur schwer unterscheiden. Wir wollten deshalb für die Verordner vernünftige Orientierungshilfen erarbeiten.

Das zweite Faktum ist unser Interesse, pflanzliche Arzneimittel für die Versicherten auch weiterhin verordnungsfähig zu erhalten.
Und da wir wissen, wie häufig diese Medikamente von den Versicherten auch ohne Rezept in der Apotheke direkt gekauft werden, wollen wir, dass auch die OTC-Käufer möglichst nur Mittel mit einer guten Qualität bekommen. Daher fühlen wir uns als Krankenkasse aufgerufen, Informationen zu erarbeiten, die eine sinnvolle Auswahl dieser Mittel in der Apotheke erleichtern.

Worin unterscheidet sich Ihr Modellvorhaben von den zahlreichen anderen Modellversuchen, die es gegenwärtig in der Bundesrepublik Deutschland gibt?

Glaeske: Die meisten anderen Modellversuche beschränken sich in ihrer Fragestellung auf eine Therapierichtung (z. B. die Homöopathie) oder auf ein bestimmtes Krankheitsbild (z. B. Diabetes mellitus). Wir haben dagegen ein Modellvorhaben geplant, in dem niedergelassene Vertragsärzte im Rahmen ihrer üblichen Patientenversorgung die Möglichkeit bekommen, je nach Indikation frei auszuwählen, wie sie therapieren wollen - ob sie beispielsweise ein Phytopharmakon oder ein Medikament mit einem chemisch-synthetischen Inhaltsstoff verordnen. Das heisst, der gleiche Arzt kann eine schulmedizinische oder eine komplementärmedizinische Methode anwenden.

Dazu muss er allerdings soweit kompetent sein, dass er jeweils qualifiziert entscheiden kann, welche Methode im Einzelfall die sinnvollere ist. Insofern machen wir hier also keinen «Binnenversuch», der ausschliesslich eine Therapierichtung prüft, sondern versuchen die Integration verschiedener Therapiemethoden zu evaluieren.

Dazu kommt noch, dass bei der Wahl der Therapiemethode nicht nur die Indikation, sondern auch die Patientenpräferenzen berücksichtigt werden sollen. Das wird sich sicher positiv auf die Patienten-Compliance auswirken und uns daher auch ermöglichen, valide zu beurteilen, wie sich die Anwendung komplementärmedizinischer Methoden nicht nur im Hinblick auf die Behandlungsergebnisse sondern auch in bezug auf die Wirtschaftlichkeit der Therapie auswirken. Diese Frage ist ein Zielparameter des Modellvorhabens.

\section{Welche Ärzte werden an dem Modellvorhaben teilnehmen?}

Glaeske: Wir rechnen mit etwa 200 Ärzten, die im Sinne einer ganzheitlich orientierten Medizin bzw. Komplementärmedizin unterschiedliche Methoden anwenden und bereit sind, die Auswahl jeweils zusammen mit dem Patienten vorzunehmen.

\section{KARGER


Phytotherapie ist aber eigentlich keine Komplementärmedizin, sondern - allein von ihrer historischen Entwicklung her - genuiner Bestandteil der regulären Medizin.

Glaeske: Dem stimme ich zu. Ich bin absolut der Meinung, dass die Zuordnung der Phytotherapie zu den besonderen Therapierichtungen - mit der man dieser Methode einen Gefallen tun wollte - etwas sehr Problematisches war. Es hat sie in den letzten 20 Jahren eher negativ beeinflusst. Manche Hersteller haben sich nämlich unter dem Etikett «besondere Therapierichtungen» sicher gefühlt und für ihre Präparate nichts mehr getan. Es ist aber eine Notwendigkeit, dass bei pflanzlichen Präparaten, genauso wie bei den chemisch-synthetischen Arzneimitteln, die wissenschaftliche Erkenntnis fortschreitet.

Gerade im Bereich der Phytopharmaka wurden in den letzten Jahren erhebliche Erkenntnisfortschritte erzielt. Sie machen es möglich, auch für diese Medikamente auf eine wissenschaftlich adäquate Weise den Wirksamkeitsnachweis zu führen. Und den fordern wir als Krankenkasse auch. Wir sind der Meinung, dass es heute absolut kontraproduktiv ist, sich hier auf den Schutz, den der Gesetzgeber für besondere Therapierichtungen vorgesehen hat, zurückzuziehen.

Insofern haben wir im Rahmen unseres Modellvorhabens auch die Qualitätskriterien und den Wirksamkeitsnachweis für Phytopharmaka zu einem der Schwerpunkte gemacht.

Zusätzlich wollen wir auf diese Weise auch jene Firmen «honorieren», die sich in den letzten Jahren um eine Qualitätssicherung und wissenschaftliche Entwicklung von Phytopharmaka bemüht haben.

Ist das der Grund, warum Sie auch im wissenschaftlichen Beirat des neugegründeten Komitees «Forschung Naturmedizin (KFN)» tätig sind?

Glaeske: Ja. Das KFN hat es sich zur Aufgabe gemacht, die Öffentlichkeit einschliesslich der Fachkreise über die Ergebnisse der Forschungsanstrengungen im Rahmen der Phytotherapie zu informieren. Die forschenden Phythopharmaka-Hersteller tragen hier ihre wissenschaftlichen Ergebnisse zusammen, um sie einem möglichst breiten Publikum zugänglich zu machen. Es geht hier wie in unserem Modellvorhaben darum, eine differenzierte Sicht der Phyto- therapie zu fördern und Ansprüche an eine gute Qualität zu formulieren.

Ist man wirklich bereit, Ross und Reiter zu nennen, wird also die Öffentlichkeit konkret erfahren können, welche Präparate und welche Firmen Qualität bieten?

Glaeske: Der Arzneimittelmarkt funktioniert im Bereich der Phytotherapie nicht wie bei wirkstoffgleichen Generika, so dass es nicht ausreicht, beispielsweise bei leichten und mittelschweren Depressionen einfach nur Johanniskrautextrakte zu empfehlen. Bei der Beurteilung der Qualität eines konkreten pflanzlichen Präparates $-\mathrm{z}$. B. eines Johanniskrautextrakts - ist es wichtig zu wissen, wie der Extrakt hergestellt wird, welche Dosierung er beinhaltet, welche Belege es für seine Wirksamkeit gibt usw. Diese Parameter können nämlich bei verschiedenen Johanniskrautextrakten - um bei diesem Beispiel zu bleiben - sehr unterschiedlich sein. Das gilt natürlich für alle Drogen. Man wird also überhaupt nicht umhin kommen, Präparatenamen und Hersteller zu nennen, wenn man therapierelevante Aussagen machen und im Rahmen unseres Modellvorhabens konkrete Empfehlungen abgeben will.

Auf welche Weise wird das Modellvorhaben wissenschaftlich evaluiert?

Glaeske: Es ist vorgesehen, die Versorgung der Patienten wissenschaftlich zu begleiten und die Evaluationsergebnisse in jedem Falle zu publizieren. Wir betreiben dabei eine wirkliche Versorgungsforschung, denn wir schaffen hier eine Möglichkeit, Daten über die reale Versorgung von Patienten analysieren zu können. Im Mittelpunkt der Evaluation stehen also die Wirksamkeit der Behandlung, die Wirtschaftlichkeit und die Lebensqualität des Patienten.

\section{Hinweis}

Die Dokumentation der Expertenkommission BPI/Barmer Ersatzkasse zum Modellvorhaben zur Förderung der Verordnung von pflanzlichen Arzneimitteln, Homöopathika und anthroposophischen Arzneimitteln gemäß SGB V wird im 1. Quartal $2000 \mathrm{im}$ Verlag S. Karger GmbH, Freiburg, erscheinen. 